\title{
Sheep milk kefir sweetened with different sugars: Sensory acceptance and consumer emotion profiling
}

\author{
Cristiane P. Larosa, ${ }^{1}$ Celso F. Balthazar, ${ }^{2}$ Jonas T. Guimarâes, ${ }^{2}$ Ramon S. Rocha, ${ }^{1,2}$ Ramon Silva, ${ }^{1,2}$ \\ Tatiana C. Pimentel, ${ }^{3}$ Daniel Granato, ${ }^{4}$ Maria Carmela K. H. Duarte, ${ }^{2}$ Marcia C. Silva, ${ }^{1}$ Mônica Q. Freitas, ${ }^{1}$ \\ Adriano G. Cruz, ${ }^{1 *}$ and Erick A. Esmerino ${ }^{2}$ \\ ${ }^{1}$ Departamento de Alimentos, Instituto Federal de Educação, Ciência e Tecnologia do Rio de Janeiro (IFRJ), 20270-021, Rio de Janeiro, Brazil \\ ${ }^{2}$ Faculdade de Veterinária, Universidade Federal Fluminense (UFF), 24230-340 Niterói, Rio de Janeiro, Brazil \\ ${ }^{3}$ Instituto Federal de Educação, Ciência e Tecnologia Paraná (IFPR), Paranavaí, Paraná, 87703-536, Brazil \\ ${ }^{4}$ Food Processing and Quality, Production Systems Unit, Natural Resources Institute Finland (Luke), FI-02150 Espoo, Finland
}

\begin{abstract}
The objective of this study was to determine the sensory acceptance and emotional profile of sheep milk kefir sweetened with different sugars (demerara sugar, brown sugar, fructose, coconut sugar, and honey, 100 $\mathrm{g} / \mathrm{L})$. Consumers $(\mathrm{n}=100)$ assessed sensory acceptance (appearance, aroma, taste, texture, and overall impression), and expressed their emotions (satisfied, active, loving, calm, comfortable, energetic, happy, healthy, refreshing, disgusted, worried, and upset). The emotions "satisfied," "active," "comfortable," "energetic," "healthy," and "refreshing" were found between moderate and very high levels, indicating that they are important emotions for the characterization and sensory acceptance of kefir samples. The use of different sugars had no influence on the intensity of the emotions "calm," "comfortable," "happy," or "disgusted," and resulted in a greater sense of satisfaction. The use of demerara sugar or fructose did not alter the acceptance of the products or the intensity of emotions. The use of brown sugar decreased acceptance (taste, texture, and overall impression) and the intensity of the emotions "active," "loving," "energetic," "healthy," and "refreshing." The use of coconut sugar decreased acceptance (appearance, aroma, and taste) and the intensity of the emotions "refreshing" and "upset." The use of honey improved acceptance in appearance and aroma but reduced the intensity of the emotions "active," "loving," "energetic," and "healthy." Based on sensory data, it is recommended to use demerara sugar or fructose as a substitute for sucrose. In conclusion, the study of emo-
\end{abstract}

Received April 11, 2020.

Accepted August 24, 2020.

*Corresponding author: food@globo.com tions can be used as an additional tool for obtaining data related to the sensory acceptance of products.

Key words: sheep milk, kefir, sugar, sensory acceptance, emotion profiling

\section{INTRODUCTION}

Kefir is a fermented milk product prepared from the fermentation of milk (cow, goat, camel, sheep, or buffalo) by heterolactic microorganisms present in kefir grains or commercial starter cultures (Mitra and Ghosh, 2020). Kefir consumption is associated with several health benefits (Bourrie et al., 2018; Wang et al., 2019). In recent years, there has been a greater demand for products in which sucrose is replaced by sugars with greater nutritional value and possible beneficial health effects (Asghar et al., 2019). The most studied nonconventional sugars are demerara sugar (obtained from semirefined sugar cane; Curi et al., 2017), brown sugar (obtained from unrefined sugar cane; Curi et al., 2017), sugar from coconut sap (Asghar et al., 2019), honey (produced by bees; Nascimento and Mondal, 2017), and fructose (present in fruits; Nascimento and Mondal, 2017).

Worldwide, cow milk is the most consumed milk, and presented a production of 683.2 million tons in 2018 . Thus, $81 \%$ of the world milk production is derived from cattle, followed by milks from other species, such as buffalo (15.1\%), goat $(2.2 \%)$, sheep $(1.3 \%)$, and camel $(0.4 \%$; FAO, 2020). Sheep milk consumption is at 10.6 million t/yr, and the population of sheep is at 1.2 billion (Cannas et al., 2019; FAO, 2020). The market for sheep milk dairy products has increased due to the quality of the products, high yield, and nutritional value, as sheep milk presents higher concentrations of fats, proteins, vitamins, and minerals compared with the milks of other domestic species (Balthazar et al., 2017). Sheep milk is commonly used to produce fine cheeses, yogurts, 
and beverages (Balthazar et al., 2019), and its use as raw material for kefir processing could be an interesting alternative.

Regardless of the health benefits that a product may provide, its sensory acceptance is a determining factor for its inclusion in the daily diet (O'Brien et al., 2017). However, there is no consensus that high sensory acceptance is sufficient to predict a product's commercialization potential or its life cycle and success in the market (Jiang et al., 2014). The positive emotions evoked by food products can increase the pleasure of consumption, promote their purchase, and dominate the decision to repurchase and include them in the regular diet (Canetti et al., 2002). In addition, specific emotions can be a critical factor in differentiating food products that have similar characteristics, prices, and packaging (Jiang et al., 2014). Studies involving the relationship between emotions evoked during consumption and sensory acceptance or discrimination of food products are still scarce (Jiang et al., 2014). Nonetheless, none of these studies have explored the analysis of kefir sweetened with different sugars. Following the technological need of new sheep milk-based foods, the aim of this study was to determine sensory acceptance and to explore consumers' emotions about sheep milk-based kefir sweetened with different sugars (demerara sugar, brown sugar, fructose, coconut sugar, and honey).

\section{MATERIALS AND METHODS}

\section{Kefir Manufacture}

Frozen pasteurized sheep milk $\left(-18^{\circ} \mathrm{C}\right)$ was combined with skim milk powder $(35 \mathrm{~g} / \mathrm{L})$ and $100 \mathrm{~g} / \mathrm{L}$ of sugars. The mixture was heat-treated in a water bath at $90^{\circ} \mathrm{C}$ for $3 \mathrm{~min}$ and cooled to $25^{\circ} \mathrm{C}$. Then, $10 \mathrm{~mL} / \mathrm{L}$ of the activated $\left(1 \mathrm{~g} / 100 \mathrm{~mL}, 24 \mathrm{~h}, 25^{\circ} \mathrm{C}\right.$ ) starter culture (Lactococcus lactis ssp. lactis, Lactococcus lactis ssp. lactis biovar diacetylactis, Lactobacillus brevis, Leuconostoc spp., and Saccharomyces bayanus, Lyofast MT 030 LV, Sacco, Campinas, Brazil) were added, and the mixture was fermented at $25^{\circ} \mathrm{C}$ for $24 \mathrm{~h}$ (Montanuci et al., 2012). Thereafter, the kefir samples were homogenized and stored in polystyrene bottles for $1 \mathrm{~d}$. The kefir formulations were prepared in 3 batches $(10 \mathrm{~L}$ each), one after the other. Six kefir formulations were prepared with different sweeteners: sucrose (SUC), demerara sugar (DEM), brown sugar (BSG), fructose (FRU), coconut sugar (COC), and honey $(\mathbf{H O N})$.

\section{Sensory Acceptance}

Consumers were recruited through personal contact and invitations via social media, and individuals who were healthy, consumed fermented dairy products, had already consumed sheep milk or sheep milk dairy products, and had available time for the sensory analyses were selected. One hundred regular consumers of fermented dairy products ( $45 \%$ men, $55 \%$ women; aged 18-64 years; mean age: 26 years) participated in the sensory acceptance test. All of the participants consumed yogurts, fermented milks, or fermented whey beverages at least 3 times a week.

Sensory analyses were performed in individual sensory cabins located at the Food Department of the Federal Institute of Rio de Janeiro (Rio de Janeiro, Brazil). Kefir samples $(30 \mathrm{~mL})$ were randomly coded and served in a random and monadic manner. Consumers received the 6 formulations one at a time, and they assessed product acceptance (appearance, aroma, taste, texture, and overall impression) using a 9-point hedonic scale (García-Gómez et al., 2019; Fidelis et al., 2020). Then, the consumers received the 6 formulations again, one at a time, and they were asked to express their physical feelings or psychological emotions using 12 terms, of which 9 were positive (satisfied, active, loving, calm, comfortable, energetic, happy, healthy, and refreshing) and 3 negative (disgusted, worried, and upset), using a 5 -point scale $(1=$ nothing, $2=$ slightly, $3=$ moderately, $4=$ very, and $5=$ extremely, O'Brien et al., 2017). A glass of water and biscuits were provided for cleansing the palate during evaluation of the different kefir formulations.

\section{Statistical Analyses}

Data were submitted to one-way ANOVA, and Tukey's test was used to compare the means $(P<$ $0.05)$. Principal component analysis was performed using a matrix of 6 rows (treatments) and 12 columns (number of emotions) with confidence ellipses using the bootstrapping technique with 500 resampling, meaning 500 virtual panels were built. Analysis was performed in XLSTAT 2020 software (Addinsoft, Paris, France).

\section{RESULTS AND DISCUSSION}

\section{Sensory Acceptance}

Sensory acceptance data are shown in Figure 1. The mean acceptance scores of kefir samples were between 5.03 and 8.45 for appearance, aroma, taste, texture, and overall impression, indicating that consumers were indifferent to some formulations and very fond of others. The use of DEM or FRU resulted in similar sensory acceptance $(P>0.05)$ compared with SUC. Thus, from the sensory standpoint, it is possible to replace sucrose with demerara or fructose. 


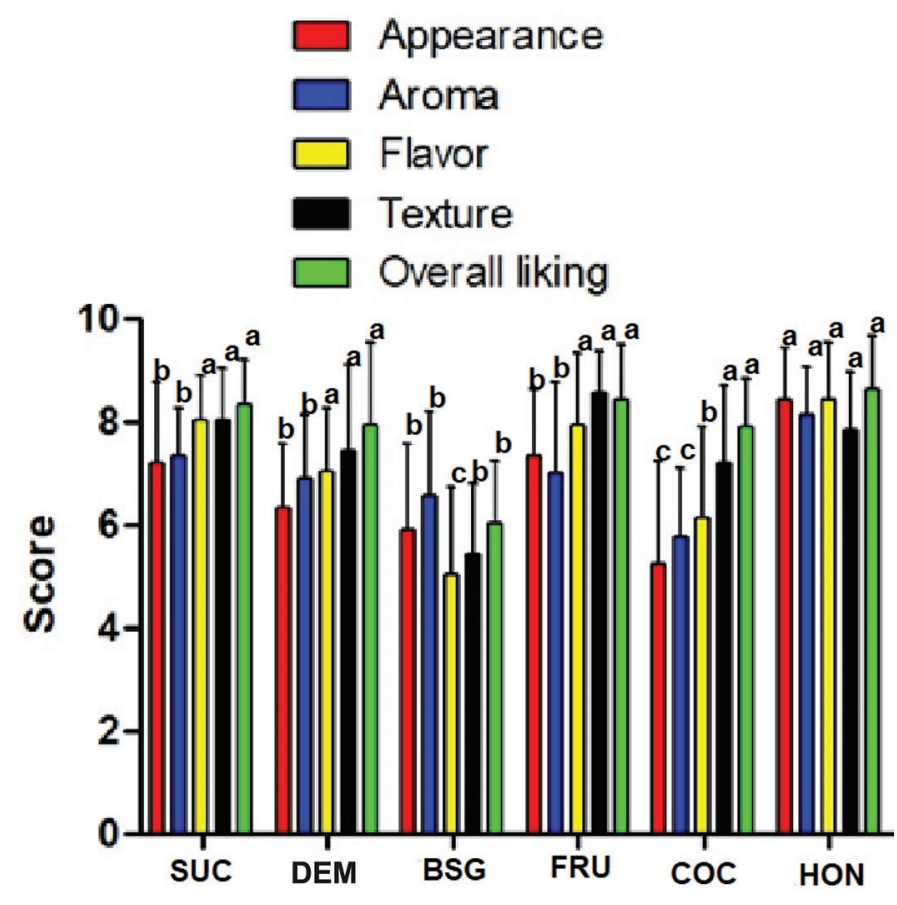

Figure 1. Sensory acceptance (appearance, aroma, flavor, texture, and overall liking) of experimental kefir fermented milk formulations. Values are expressed as mean $\pm \mathrm{SD}\left(\mathrm{n}=100\right.$ consumers). ${ }^{\mathrm{a}-\mathrm{c}}$ The same lowercase letters indicate lack of statistical difference $(P>0.05)$ for the same sensory attribute. $\mathrm{SUC}=$ sucrose, $\mathrm{DEM}=$ raw demerara sugar, BSG = brown sugar, FRU = fructose, $\mathrm{COC}=$ coconut sugar, $\mathrm{HON}=$ honey.

Use of BSG decreased acceptance of the products (taste and texture), with a consequent decrease in the overall impression $(P<0.05)$ compared with SUC. Decreased acceptance of taste may be related to the greater acidity of the products with BSG compared with SUC ( $\mathrm{pH} 4.33$ vs. 4.62). In fact, the pronounced acidic taste in kefir is the main negative attribute cited by consumers. Therefore, products with less acidity are generally more accepted by consumers (O'Brien et al., 2017). Products with a lower $\mathrm{pH}$ and greater acidity generally have higher viscosity, because the greater acidity leads to contraction of the casein micelle, which, in turn, results in a firmer and more cohesive structure (Montanuci et al., 2012). Thus, the greater acidity and firmness of products with added BSG contributed negatively to consumer acceptance of the products. Use of COC decreased acceptance of appearance, aroma, and taste $(P<0.05)$ but had no influence $(P>0.05)$ on the overall impression. Coconut sugar is a natural ingredient obtained from coconut tree sap, marketed in the form of large crystals with a brownish color (Curi et al., 2017). Its brownish color may be the factor responsible for decreased acceptance of appearance for our COC kefir. The decrease in taste may be related to the greater acidity of the products with added COC compared with SUC (pH 4.35 vs. 4.62). The higher concentration of Lactobacillus (8.69 vs. $7.73 \log \mathrm{cfu} / \mathrm{g}$ ) may have resulted in the formation of aromatic compounds that contributed negatively to the aroma. However, lower acceptance of the appearance, aroma, and taste did not decrease the overall impression of the products.

Finally, use of honey increased $(P<0.05)$ the mean scores of appearance and aroma but had no influence $(P>0.05)$ on the overall impression of our HON kefir. The use of honey in kefir can make the product more yellowish (Coskun and Karabulut Dirican, 2019). In addition, adding honey to kefir formulations may enhance the formation of acetaldehyde and aromatic compounds during fermentation, in which case the end product may contain floral and fruity aromas (Fiorda et al., 2016). Thus, the more yellowish color and the possible improvement in the aroma compounds may have contributed positively to the acceptance of HON kefir.

\section{Emotional Profiles}

The emotions evoked by the kefir samples sweetened with different sugars are shown in Table 1. Results showed that kefir samples evoked the analyzed emotions differently in terms of intensities. The attributes "loving," "calm," "happy," "disgust," "worried," and "upset" were observed in levels between "absent" and "slight" (values between 1 and 2), indicating that they were not associated with the kefir samples. It is important to mention that the low values attributed to negative emotions (disgust, worried, and upset) suggest that consumers did not dislike kefir samples (O'Brien et al., 2017). The attributes "satisfied," "active," "comfortable," "energetic," "healthy," and "refreshing" were between "moderate" and "very high" levels (values between 3 and 4), indicating that they are important attributes. In fact, kefir samples are characterized as beverages with an acidic taste, a mild aroma of fresh yeast, natural effervescence, and a slightly alcoholic sensation (Montanuci et al., 2012). In addition, they are recognized as being healthy (O'Brien et al., 2017).

The use of different sugars had no influence $(P>$ $0.05)$ on the intensity of the attributes "calm," "comfortable," "happy," or "disgusted," with an increase in the intensity of "concerned" and "satisfied" $(P<0.05$, except for "satisfied" for DEM) observed. Sucrose is the main sweetener agent used in the food industry, as it does not present bitter or residual taste (Costa et al., 2019). Consumers are increasingly concerned with health, and, thus, the interest in consuming healthy foods is a current issue. In this context, there is demand to replace sucrose with healthier sugars (Curi et al., 


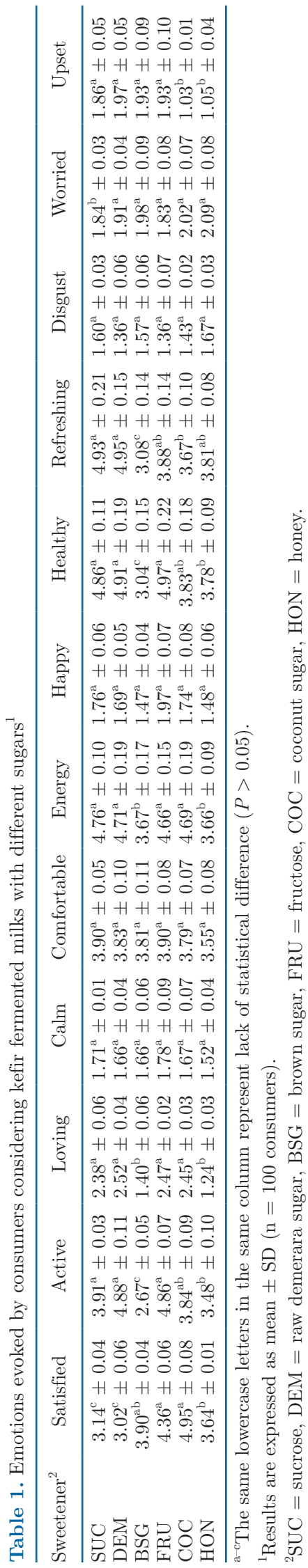

2017). The substitution of sucrose for different sugars may have altered the profile of sweet taste, which may have caused slight concern among consumers (increase in values from 1.84 to $1.91-2.09$ ) but significantly contributed to the increase in satisfaction (increase in values from 3.14 to $3.64-4.95$ ).

The use of either DEM or FRU resulted in similar $(P$ $>0.05)$ emotions compared with SUC. These results corroborate the sensory acceptance test. In turn, the use of BSG decreased $(P<0.05)$ the intensity of the attributes "active," "loving," "energetic," "healthy," and "refreshing." Kefir sweetened with BSG garnered lower mean acceptance scores for taste, texture, and overall impression (Figure 1). Possibly, the greater acidity and viscosity of this product resulted in lower intensities for the attributes "energy" and "refreshment." Similarly, the use of $\mathrm{COC}$ decreased $(P<0.05)$ the intensity of "refreshing" and "upset." The results corroborate those observed for sensory acceptance (Figure 1), in which kefir sweetened with COC garnered lower $(P<$ 0.05) mean acceptance scores for appearance, aroma, and taste. The greater acidity of this sample may have decreased the feeling of freshness. The use of honey in kefir decreased $(P<0.05)$ the intensity of the attributes "active," "loving," "energetic," "healthy," and "upset." The positive effects of HON kefir observed on the acceptance of its appearance and aroma could not be observed in the assessment of emotions evoked during consumption. On the contrary, HON showed lower intensity of several emotional attributes. Emotions that weakly correlate with the sensory acceptance of a product are of special interest, as they transmit undisclosed information about the product formulation. Thus, although HON kefir presented a higher acceptance (appearance and aroma), it evoked less intense emotion (active, loving, energetic, healthy, and upset) compared with our SUC kefir.

Figure 2 shows the principal component analysis bidimensional map, with confidence ellipses, in which are reported $79.16 \%$ of the data variability, with 61.83 and $17.33 \%$ at the first and second dimensions, respectively. Overall, 2 groups clearly exist: the first group (BSG, $\mathrm{COC}$, and HON) associated with the emotions "satisfied," "worried," and "disgust," and the second (SUC, DEM, and FRU) associated with the emotions "refreshing," "upset," "healthy," "active," "energy," "comfortable," "loving," "happy," and "calm." Confidence ellipses indicate that the SUC and DEM samples strongly overlapped; the SUC and FRU, and SUC and BSG samples slightly overlapped; and SUC was well differentiated from $\mathrm{COC}$ and HON. These findings are interesting, as they give useful information to sheep milk processors, to establish different marketing strategies for each group of samples, serving as initial guidelines. 

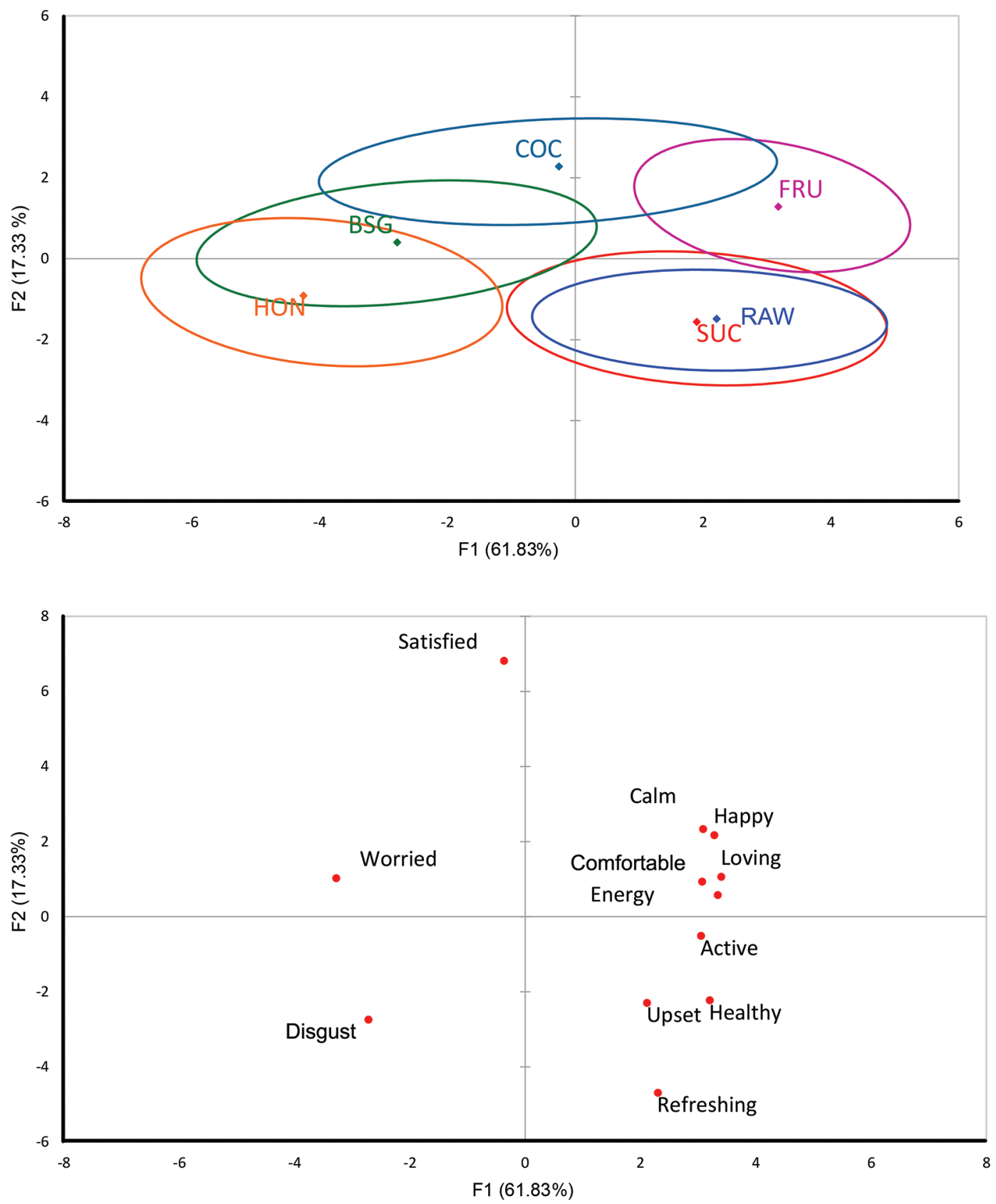

Figure 2. Representation of the kefir sample formulations and the emotional attributes in bidimensional maps of the principal component analysis from emotional questionnaire. $\mathrm{SUC}=$ sucrose, $\mathrm{DEM}=$ raw demerara sugar, $\mathrm{BSG}=$ brown sugar, $\mathrm{FRU}=$ fructose, $\mathrm{COC}=$ coconut sugar, HON = honey, F1 and F2 = first and second dimensions, respectively.

\section{CONCLUSIONS}

The results of the present study are relevant for the sheep milk dairy industry, as they indicate that emotional perceptions and sensory acceptance of kefir sweetened with different agents are directly correlated. Kefir samples with higher sensory acceptance scores were associated with higher intensities of "satisfied," "active," "comfortable," "energetic," "healthy," and "refreshing" feelings. Based on sensory characteristics, we recommend using demerara sugar or fructose as a substitute for sucrose. The evaluation of emotions evoked by products can be an important tool to obtain additional information that can be used for product optimization and market strategies by the sheep milk industry. 


\section{ACKNOWLEDGMENTS}

This study was financed in part by the Coordenação de Aperfeiçoamento de Pessoal de Nível Superior, Brasil (CAPES), Finance Code 001. The authors are grateful to the Conselho Nacional de Pesquisa Científica (CNPQ), Fundação de Amparo a Pesquisa no Rio de Janeiro (FAPERJ), and the Instituto Federal de Educação, Ciência e Tecnologia do Rio de Janeiro (IFRJ), Brazil, for financial support. The authors have not stated any conflicts of interest.

\section{REFERENCES}

Asghar, M. T., Y. A. Yusof, M. N. Mokhtar, M. E. Ya'acob, H. M. Ghazali, L. S. Chang, and Y. N. Manaf. 2019. Coconut (Cocos nucifera L.) sap as a potential source of sugar: Antioxidant and nutritional properties. Food Sci. Nutr. https://doi.org/10.1002/ fsn3.1191.

Balthazar, C. F., T. C. Pimentel, L. L. Ferrão, C. N. Almada, A. Santillo, M. Albenzio, N. Mollakhalili, A. M. Mortazavian, J. S. Nascimento, M. C. Silva, M. Q. Freitas, A. S. Sant'Ana, D. Granato, and A. G. Cruz. 2017. Sheep milk: Physicochemical characteristics and relevance for functional food development. Compr. Rev. Food Sci. Food Saf. 16:247-262. https://doi.org/10.1111/1541-4337 .12250 .

Balthazar, C. F., A. Santillo, J. T. Guimarães, V. Capozzi, P. Russo, M. Caroprese, R. Marino, E. A. Esmerino, R. S. L. Raices, M. C. Silva, H. L. A. Silva, M. Q. Freitas, D. Granato, A. G. Cruz, and M. Albenzio. 2019. Novel milk-juice beverage with fermented sheep milk and strawberry (Fragaria $\times$ ananassa): Nutritional and functional characterization. J. Dairy Sci. 102:10724-10736. https:/ /doi.org/10.3168/jds.2019-16909.

Bourrie, B. C., P. D. Cotter, and B. P. Willing. 2018. Traditional kefir reduces weight gain and improves plasma and liver lipid profiles more successfully than a commercial equivalent in a mouse model of obesity. J. Funct. Foods 46:29-37. https://doi.org/10.1016/j.jff 2018.04.039.

Canetti, L., E. Bachar, and E. M. Berry. 2002. Food and emotion. Behav. Processes 60:157-164. https://doi.org/10.1016/S0376 -6357(02)00082-7.

Cannas, A., L. O. Tedeschi, A. S. Atzori, and M. F. Lunesu. 2019. How can nutrition models increase the production efficiency of sheep and goat operations? Anim. Front. 9:33-44. https://doi.org/ 10.1093/af/vfz005.

Coskun, F., and L. Karabulut Dirican. 2019. Effects of pine honey on the physicochemical, microbiological and sensory properties of probiotic yoghurt. Food Sci. Technol. (Campinas) 39(Suppl. 2):616-625. https://doi.org/10.1590/fst.24818.
Costa, G. M., M. M. Paula, C. E. Barão, S. J. Klososki, E. G. Bonafé, J. V. Visentainer, A. G. Cruz, and T. C. Pimentel. 2019. Yoghurt added with Lactobacillus casei and sweetened with natural sweeteners and/or prebiotics: Implications on quality parameters and probiotic survival. Int. Dairy J. 97:139-148. https://doi.org/10 .1016/j.idairyj.2019.05.007.

Curi, P. N., C. D. S. Carvalho, D. L. Salgado, R. Pio, M. Pasqual, F. B. M. D. Souza, and V. R. D. Souza. 2017. Influence of different types of sugars in physalis jellies. Food Sci. Technol. (Campinas) 37:349-355. https://doi.org/10.1590/1678-457x.08816.

FAO. 2020. FAOSTAT: Compare data. Food and Agriculture Organization of the United Nations. Accessed Jun. 12, 2020. http:// faostat.fao.org/.

Fidelis, M., S. M. De Oliveira, J. S. Santos, G. B. Escher, R. S. Rocha, A. G. Crus, M. A. V. Do Carmo, L. Azevedo, T. Kaneshima, W Y. Oh, F. Shahidi, and D. Granato. 2020. From byproduct to a functional ingredient: Camu-camu (Myrciaria dubia) seed extract as an antioxidant agent in a yogurt model. J. Dairy Sci. 103:11311140. https://doi.org/10.3168/jds.2019-17173.

Fiorda, F. A., G. V. de Melo Pereira, V. Thomaz-Soccol, A. P. Medeiros, S. K. Rakshit, and C. R. Soccol. 2016. Development of kefir-based probiotic beverages with DNA protection and antioxidant activities using soybean hydrolyzed extract, colostrum and honey. Lebensm. Wiss. Technol. 68:690-697. https://doi.org/10 .1016/j.lwt.2016.01.003.

García-Gómez, B., A. Romero-Rodríguez, L. Vázquez-Odériz, N. Muñoz-Ferreiro, and M. Vázquez. 2019. Sensory quality and consumer acceptance of skim yoghurt produced with transglutaminase at pilot plant scale. Int. J. Dairy Technol. 72:388-394.

Jiang, Y., J. M. King, and W. Prinyawiwatkul. 2014. A review of measurement and relationships between food, eating behavior and emotion. Trends Food Sci. Technol. 36:15-28. https://doi.org/10 $.1016 / j . t i f s .2013 .12 .005$

Mitra, S., and B. C. Ghosh. 2020. Quality characteristics of kefir as a carrier for probiotic Lactobacillus rhamnosus GG. Int. J. Dairy Technol. 73:384-391. https://doi.org/10.1111/1471-0307.12664.

Montanuci, F. D., T. C. Pimentel, S. Garcia, and S. H. Prudencio. 2012. Effect of starter culture and inulin addition on microbial viability, texture, and chemical characteristics of whole or skim milk Kefir. Food Sci. Technol. (Campinas) 32:850-865. https://doi.org/ 10.1590/S0101-20612012005000119.

Nascimento, M., and A. Mondal. 2017. Microbial ecology of confectionary products, honey, sugar, and syrups. Pages 533-546 in Quantitative Microbiology in Food Processing: Modeling the Microbial Ecology. John Wiley and Sons, Chichester, UK.

O'Brien, K., C. Boeneke, W. Prinyawiwatkul, J. Lisano, D. Shackelford, K. Reeves, M. Christensen, R. Hayward, K. C. Ordonez, and L. K. Stewart. 2017. Sensory analysis of a kefir product designed for active cancer survivors. J. Dairy Sci. 100:4349-4353. https:// doi.org/10.3168/jds.2016-12320. 